\title{
La apuesta brasileña en la Antártida: \\ trayectoria reciente y perspectivas futuras a la luz de la inauguración de la nueva Estación Antártica Comandante Ferraz
}

\section{Resumen}

Ignacio Javier Cardone*

La inauguración de la nueva estación Antártica Comandante Ferraz y la intención de adquirir un nuevo navío de apoyo logístico parecen indicar un interés renovado de Brasil en su política antártica. Sin embargo, resulta necesario evaluar la trayectoria reciente y la evolución de otros factores para obtener una lectura más contextualizada. Para ello, el presente artículo analiza la evolución reciente del programa antártico brasileño en base a tres factores: infraestructura, estructura institucional y de investigación científica. Así, podemos observar que la fuerte inversión en infraestructura física ha fortaleciendo el perfil preexistente, pero sin incorporar nuevas capacidades. Asimismo, la estructura institucional del programa antártico brasileño continúa siendo relativamente reducida y poco especializada. En términos de las investigaciones científicas, Brasil ha experimentado un proceso de institucionalización relevante, pero aún no ha conseguido alcanzar un nivel de estabilidad, y la exclusión de las humanidades y ciencias sociales lo limita en un aspecto que va adquiriendo creciente importancia a nivel mundial. Lo anterior sugiere que Brasil ha sido exitoso en mantener sus operaciones antárticas en un nivel de importancia internacional, pero no se espera que experimente una expansión o incremento de peso significativo.

Palabras Clave: política antártica brasileña - PROANTAR - Estación Antártica Comandante Ferraz Brasil - Antártida

\section{Brazil's commitment to Antarctica: recent trajectory and future prospects in light of the inauguration of the new Comandante Ferraz Antarctic Station}

\begin{abstract}
The recent inauguration of the rebuilt Brazilian Antarctic Station Comandante Ferraz and the announcement of the acquisition of a new polar ship seem to indicate a renewed interest of Brazil in Antarctica. However, a more contextualized interpretation of those events demands an appraisal of the recent evolution of other factors. Following that aim, the present paper presents an analysis of the recent evolution of three factors of the Brazilian Antarctic programme: infrastructure and political and scientific institutional setting. The analysis concludes that a strong investment in physical infrastructure have strengthened the pre-existent profile of Brazil in Antarctica, but have not added new capacities. Regarding the political institutional setting, it continues to be relatively reduced in scale and poorly specialized. At last, the scientific research has experienced a relevant process of institutionalization, but without reaching stability in terms of funding and influence within the Brazilian Antarctic politics. Furthermore, the exclusion of humanities and social sciences presents a relevant limitation in a field that is acquiring international prominence. Therefore, Brazil have been successful in maintaining its Antarctic involvement at a relevant scale internationally, but it is not expected that would expand significantly in the near future.
\end{abstract}

Key words: Brazilian Antarctic Politics - PROANTAR - Comandante Ferraz Antarctic Station - Brazil Antarctica

TRABAJO RECIBIDO: 17/02/2021 TRABAJO ACEPTADO: 28/03/2021

Esta obra está bajo una licencia internacional https://creativecommons.org/licenses/by-sa/4.0/

\footnotetext{
* Doctor en Relaciones Internacionales, Universidade de São Paulo (Brasil) y el King’s College London. Investigador de post-doctorado del Núcleo de Pesquisa em Relações Internacionais (NUPRI) de la Universidade de São Paulo. Correo electrónico: ignaciocardone@ gmail.com

El autor agradece los comentarios y sugerencias de los/las dictaminadores anónimos/as de los Cuadernos de Política Exterior Argentina.
} 


\section{Introducción}

La inauguración, en enero de 2020, de la nueva Estación Antártica Comandante Ferraz (EACF), caracterizada como una de las bases más modernas y avanzadas tecnológicamente de la Antártida, se presenta como un hito que marca una apuesta renovada de Brasil en el continente blanco. Ello, sumado a la inclusión de la Antártida dentro del entorno estratégico del país (Abdenur \& Neto, 2014; Mattos, 2014) y al reciente anuncio de la intención de adquirir un nuevo navío polar de apoyo logístico parecería, en primera lectura, indicar un marcado renacimiento del interés brasileño en la región polar. Sin embargo, una mirada más atenta parece relativizar esa lectura; particularmente cuando se toma en consideración que la reconstrucción de la EACF respondió a un incendio que destruyó la anterior base en su casi totalidad y que el programa ha experimentado diversas dificultades que han amenazado la continuidad de las investigaciones en diversas oportunidades y que, incluso, se proyectan hacia el futuro ${ }^{1}$. Ello nos lleva a preguntarnos hasta qué punto podemos caracterizar a la trayectoria reciente de Brasil en la Antártida como indicativa de un renovado interés y respuesta a una política proactiva por parte del Estado brasileño. Para dar respuesta a esa pregunta, nos proponemos aquí evaluar la trayectoria reciente de Brasil en la Antártida en base a tres factores que consideramos como determinantes de su política: infraestructura, estructura institucional y de investigación científica. Ello nos permitirá evaluar los recientes eventos, incluida la inauguración de la nueva EACF, dentro de una mirada más contextualizada, permitiendo una mejor apreciación de los rumbos actuales y futuros de la política antártica de Brasil.

En términos generales, Brasil ha sido relativamente relegado en la producción académica sobre política antártica. Entre los trabajos académicos realizados en Brasil y publicados en lengua portuguesa, ha sido escasa la producción sobre la política antártica brasileña, aunque dicho número se ha incrementado durante los últimos años ${ }^{2}$. Asimismo, también han sido escasos los trabajos publicados en otros idiomas. Entre estos últimos, podemos destacar las contribuciones de Miryam Colacrai (1988, 1989), donde se analizaron las posibilidades de cooperación antártica de Brasil con otros países latinoamericanos, en especial con Argentina; la obra de Jack Child (1988), que considera a Brasil como una potencia antártica modesta, pero emergente; el trabajo de Abdenur y Neto (2014), con su análisis sobre la inclusión de la Antártida dentro del entorno estratégico de Brasil; y el capítulo de Sampaio, Cardone y Abdenur (2017), que revisa las conclusiones de Child.

Ciertamente, dicha relativa omisión de la política antártica brasileña en los estudios académicos se explica tanto por el limitado perfil de Brasil en el continente blanco, como por condiciones propias de la estructura institucional antártica brasileña y la relativa ausencia de especialistas en política antática en el país ${ }^{3}$. Así, las visiones sobre la presencia brasileña en la Antártida y su participación en el régimen internacional para dicho continente han sido limitadas a apreciaciones circunstanciales, muchas veces teñidas por el discurso oficial y por la visión tradicional ofrecida por la escuela de pensamiento geopolítico brasileña. Sin embargo, siendo Brasil una de las primeras Partes a incorporarse como miembro consultivo del Tratado Antártico luego de los 12 miembros originales ${ }^{4}$, una de las diez primeras economías del mundo ${ }^{5}$,

\footnotetext{
${ }^{1} \mathrm{El}$ actual programa de financiamiento ha sido considerado como insuficiente para cubrir las necesidades de las investigaciones en curso y no hay un panorama claro de que al concluir el plazo éste sea renovado en un volumen considerable. Ver Silveira (2019); Mesquita (2019).

${ }^{2}$ Ver, por ejemplo: Cardone (2014, 2015); Ferreira (2009); Gandra (2009, 2013); Lemertz (2015); Mattos (2014, 2015); Nascimento (2007); Pereira (2009).

${ }^{3}$ Nos referimos aquí, específicamente, a la ausencia de académicos especializados en política antártica. En términos generales, no existe en Brasil una tradición de estudios académicos sobre política antártica, aparte de los estudios surgidos de la tradición del pensamiento geopolítico brasileño ligado, principalmente, a la figura de Terezinha de Castro y su llamada Teoría de la Defrontación.

${ }^{4}$ Brasil adquirió el status consultivo en 1983 junto con la India, siendo, ambos, los miembros consultivos número 3 y 4 a ser incorporados, luego de Polonia (1977) y la República Federal de Alemania (1981), a
} 
y una potencia que ha reclamado continuamente un lugar preponderante dentro del escenario internacional, ciertamente conocer de modo más acabado las características, determinantes y condicionantes de su política antártica resulta esencial, tanto para el avance en el conocimiento de la política exterior de dicho país, como para una mejor apreciación sobre las complejidades involucradas en su política hacia el continente blanco.

En la búsqueda de constituir un aporte al respecto de la carencia apuntada anteriormente, el presente trabajo propone una mirada más matizada, contextualizada y actualizada, que permita una apreciación más sensible sobre las actitudes de Brasil hacia la Antártida y, particularmente, de sus intereses en dicha región. Para ello, este trabajo presenta un análisis cualitativo basado en datos obtenidos de fuentes primarias, fuentes secundarias, documentos oficiales y opiniones de expertos obtenidas en 2015 y 2020.

A continuación, el trabajo está estructurado en cuatro secciones. La primera sección ofrece una aproximación a la disponibilidad de infraestructura física por parte del Programa Antártico Brasileño (PROANTAR), incluyendo la inauguración de la nueva EACF, la disponibilidad e incorporación de medios de transporte, infraestructura para la investigación científica y la administración del programa, trazando un perfil actual y del futuro inmediato de las capacidades físicas y operativas del mismo. La segunda sección analiza la estructura institucional de la Política Antártica Brasileña (POLANTAR), incluyendo la normativa vigente, el diseño institucional y de proceso decisorio y las estructuras de apoyo al mismo, buscando así determinar las fortalezas, debilidades y adecuación de dicha estructura a las necesidades actuales y futuras. La tercera sección incluye una evaluación de la reciente evolución de la estructura institucional disponible para la investigación antártica, partiendo de la consideración del nivel de institucionalización de planes de largo plazo, grupos y centros especializados en investigación antártica. Por último, se presentan las conclusiones generales obtenidas a partir del análisis de los tres factores referidos, trazando el perfil actual y las perspectivas a futuro del programa brasileño, levantando consideraciones al respecto de la importancia política de tal programa para dicho país.

\section{Inversiones e infraestructura del Programa Antártico Brasileño: inversión concentrada, modernización y consolidación del perfil actual}

La inversión en infraestructura es, ciertamente, apenas una faceta de la apuesta que un país realiza en su intervención antártica. Siendo que la actividad principal en el continente blanco es la investigación científica; que el Tratado Antártico, firmado en 1959, prevé la maximización de la eficiencia en lo que respecta a la construcción y localización de bases en la región y la cooperación internacional; y que existen en la actualidad diversas posibilidades de tercerización de las operaciones logísticas antárticas, la inversión en infraestructura se presenta ya no como una necesidad del compromiso antártico de un país, sino como una elección dentro de un conjunto de opciones abiertas. Sin embargo, por su carácter de permanencia (o al menos de durabilidad) y las responsabilidades directas derivadas de su adquisición, instalación y operación, la inversión en infraestructura ciertamente refleja una apuesta de más largo alcance, brindando un horizonte de estabilidad - al menos en el campo de las posibilidades - a las operaciones que hacen usufructo de dicha infraestructura. Por ese motivo, a seguir, serán consideradas como un indicador de la situación presente, pero también de la proyección en el futuro mediato sobre las posibilidades del programa antártico brasileño.

los 12 miembros originales del Tratado: Argentina, Australia, Bélgica, Chile, Estados Unidos, Francia, Japón, Noruega, Nueva Zelandia, Reino Unido, Sudáfrica y la Unión Soviética.

5 Según datos del Banco Mundial (2019), Brasil se colocó en la $9^{\text {na }}$ posición en términos del Producto Bruto Interno en términos nominales, y en $10^{\mathrm{ma}}$ posición en términos de paridad del poder de compra. 
El PROANTAR cuenta con una infraestructura orientada exclusivamente a sus operaciones antárticas. Para la materialización de las tareas de investigación fuera del continente antártico y la administración del programa, éste utiliza la infraestructura disponible por parte de universidades, la Marina y el Ministerio de Relaciones Exteriores. Como veremos en el siguiente apartado, ello responde al diseño institucional adoptado por Brasil desde el comienzo de su involucramiento activo en Antártida, el cual definía una economía de recursos para su implementación (Exposição de Motivos N016/76, 1976). Ello ha permitido que Brasil concentre sus esfuerzos financieros de infraestructura en el equipamiento que le permite operar en la región austral.

En términos de movilidad acuática para sus operaciones antárticas, Brasil cuenta con dos navíos principales: el navío polar Almirante Maximiano y el navío de apoyo oceanográfico Ary Rongel, ambos dedicados exclusivamente para las operaciones antárticas y adquiridos con dicha finalidad ${ }^{6}$. El Almirante Maximiano fue incorporado a las operaciones brasileñas en 2009. Cuenta con capacidad para albergar unas 120 personas, cinco laboratorios (dos secos, dos húmedos y uno mixto), diversos equipamientos para el apoyo a la investigación y la realización de tareas hidrográficas y oceanográficas, y un hangar y helipuerto con capacidad para dos aeronaves; además de un moderno sistema de maniobra, navegación y comunicación. El navío es habilitado para operar en aguas con presencia de hielo, pero no es categorizado como un rompehielos (es decir, que no puede abrir camino en hielo sólido). El Ary Rongel fue incorporado a las operaciones brasileñas en 1994 y es un navío con características propias para brindar apoyo logístico. Cuenta con capacidad para 105 personas, dos laboratorios y un conteiner para el apoyo a la investigación, dos grúas de carga y descarga y un cabestrante oceanográfico, y capacidad para operar con hasta dos helicópteros; además de un moderno sistema de navegación y comunicación. Cada navío cuenta a su vez con embarcaciones propias para el desembarco y la ejecución de tareas marinas. Sumado a ello y para brindar apoyo aéreo a las operaciones embarcadas, la marina aérea contribuye con una dotación de helicópteros UH13. Dichos helicópteros están siendo reemplazados por los más modernos UH-17 (Airbus/Helibras H-135 T3) siguiendo un programa de modernización del parque de helicópteros ${ }^{7}$.

Con dicho equipamiento, durante el verano, Brasil cuenta con capacidad para el traslado de personal y apoyo a las investigaciones, pudiendo albergar en dichos navíos hasta 57 personas además de la tripulación ${ }^{8}$. Así, articulados con las operaciones de traslado aéreo a cargo de la Fuerza Aérea Brasileña, los navíos de Brasil le permiten rotar cerca de 150 científicos para la realización de sus trabajos en la región. En términos de flexibilidad operativa, la flota antártica brasileña le permite operar con seguridad en la región norte de la península antártica y otras regiones libres de placas de hielo. Ciertamente dicha limitación impide la realización de trabajos en latitudes extremas y el ingreso en diversas regiones en las cuales las condiciones de hielo impiden la navegación de los navíos brasileños, pero ello no ha impedido que grupos de científicos brasileños realicen labores valiéndose de la colaboración de otros países o la contratación de empresas privadas especializadas en servicios de logística antártica.

\footnotetext{
${ }^{6}$ Todas las informaciones técnicas sobre la infraestructura del PROANTAR fueron extraídas del sitio oficial de la Secretaría da Comissão Interministerial para los Recursos del Mar (SECIRM), encargada del programa antártico brasileño: https://www.marinha.mil.br/secirm/. Consulta: 23 de agosto de 2020

${ }^{7}$ Hasta la fecha de elaboración del presente trabajo (septiembre 2020), dos de dichas aeronaves ya fueron incorporadas al $1^{\text {er }}$ Escuadrón de Helicópteros de Empleo General, que se desempeña en las operaciones aéreas del PROANTAR. Ver: https://www.marinha.mil.br/noticias/marinha-do-brasil-recebe-segundaaeronave-uh-17-para-operacao-antartica. Consulta: 5 de septiembre de 2020

${ }^{8}$ Dicha capacidad adicional es destinada a investigadores y personal de apoyo, así como también oficiales gubernamentales, personal de intercambio de la Marina de otros países, coordinadores de la SECIRM y periodistas.
} 
Buscando la actualización de su flota, en junio de 2020, se ha establecido el llamado a ofertas para la adquisición de un navío de apoyo que pueda reemplazar el Ary Rongel, el cual debería ser construido en Brasil, con una inversión prevista de aproximadamente 130 millones de dólares americanos (750 millones de reales) ${ }^{9}$. El nuevo navío busca una mayor eficiencia operativa a partir de la incorporación de tecnología que le permita reducir la cantidad de tripulación, lo que permitiría incluir una mayor cantidad de investigadores sin perjuicio de su capacidad operativa y seguridad. También se prevé la inclusión de capacidad de hangaraje cubierto y climatizado de los helicópteros (la cual está ausente del Ary Rongel), un mejor desempeño ambiental y la mejora en la tecnología de navegación y telecomunicaciones. Si bien no está previsto que dicho navío incorpore capacidad de inserción en barreras de hielo, por lo que no habrá una expansión del teatro de operaciones, sí está previsto que las alteraciones le permitan un mayor foco en la investigación y una mayor autonomía. Sumado a ello, la determinación de su construcción en Brasil significa la adquisición de capacidades y tecnologías para la construcción de navíos aptos para las aguas polares, que hoy está ausente en Brasil y que le permitiría un grado mayor de autonomía en el futuro.

En términos de infraestructura terrestre, el PROANTAR cuenta con una estación habilitada para operar durante todo el año, la Estación Antártica Comandante Ferraz (EACF), localizada en la Isla 25 de Mayo (Rey Jorge/Waterloo), al norte de la península antártica ${ }^{10}$. Dicha estación está compuesta actualmente por 45 módulos de emergencia, que fueron instalados luego del incendio que destruyó la antigua estación brasileña en 2012, que se suman a los 3 módulos de laboratorios que sobrevivieron a dicho incendio. Los 45 módulos brindan a Brasil un área de $940 \mathrm{~m}^{2}$, incluyendo laboratorios, y capacidad para albergar hasta 66 personas (incluidos los 16 militares que operan la estación). Dichas instalaciones deberán ser removidas una vez que haya sido completado el proceso de transferencia de las operaciones a la nueva estructura.

Para reemplazar la infraestructura destruida por el incendio en 2012, Brasil inauguró, el 15 de enero de 2020, la nueva Estación Antártica Comandante Ferraz; un proyecto ambicioso con diseño innovador y tecnología de punta que busca colocar a la estación brasileña en una posición destacada dentro del desempeño ambiental y seguridad operativa en el continente blanco. La estructura cuenta con $4.500 \mathrm{~m}^{2}$ de superficie cubierta, incluyendo 19 laboratorios (14 internos y 5 externos) y capacidad para albergar hasta 64 personas (35 durante el invierno). La generación eléctrica a partir de motores diésel es complementada por una serie de aerogeneradores que, se espera, generen aproximadamente un 20 a $30 \%$ de la electricidad necesaria para el funcionamiento de la estación.

Además de la estación antártica, el PROANTAR cuenta con una estación automática de investigación remota, conocida como módulo CRIOSFERA 1, ubicada en el interior del continente, a aproximadamente $650 \mathrm{~km}$ del Polo Sur. La estación debería ser complementada por un segundo modulo automático, pero dificultades financieras del programa han impedido hasta el momento su materialización. También, el PROANTAR cuenta con diversos refugios en la región antártica y dos estaciones de apoyo en territorio brasileño, en Rio Grande do Sul y Rio de Janeiro, las cuales son utilizadas para el almacenamiento y reacondicionamiento del equipamiento polar.

Por último, el PROANTAR cuenta a su disposición la utilización de aeronaves Lockheed C-130 "Hércules" para el traslado de personal y reabastecimiento de las operaciones.

\footnotetext{
${ }^{9}$ Las informaciones sobre el nuevo navío de apoyo a las operaciones antárticas brasileñas fueron extraídas de un artículo de Danilo Olivera en el sitio del Sindicato Nacional da Indústria da Construção e Reparação Naval e Offshore (Sinaval): http://sinaval.org.br/2020/05/projeto-de-novo-navio-de-apoioantartico-segue-em-consulta/. Consulta: 23 de agosto de 2020

${ }^{10}$ La Isla 25 de mayo alberga 10 estaciones de 9 países y varios refugios, incluyendo a la base Carlini de Argentina, además de un laboratorio alemán que se encuentra dentro de la estación argentina.
} 
Dichas aeronaves pertenecen al Primer Grupo de Transporte Aéreo de la Fuerza Aérea Brasileña "Escuadrón Gordo", el cual utiliza parte de su escuadrón para dichas tareas durante el verano (aproximadamente 10 vuelos de transporte y reabastecimiento), y en invierno para el reabastecimiento por descargas aéreas. Está previsto que dichas aeronaves sean reemplazadas, a partir de 2023, siguiendo la modernización de la actual flota de C-130 por los nuevos KC-390 fabricados por la empresa de aviación brasileña EMBRAER.

En resumen, la infraestructura física del PROANTAR muestra ser de alcance limitado, pero adecuadamente dimensionada y ecualizada para los objetivos actuales de Brasil en la Antártida. En términos de su actualidad, el PROANTAR cuenta con equipos de última tecnología en casi todos los ámbitos de su actuación, destacando la calidad, tecnología de punta y diseño de la nueva EACF, y con algunos elementos importantes, como uno de sus navíos, los helicópteros y las aeronaves de transporte de carga, en proceso de modernización. Puede afirmarse que la modernización reciente de la infraestructura del PROANTAR no ha modificado significativamente las capacidades operativas del PROANTAR ni su alcance, en el sentido de que ni la nueva estación, ni la adquisición del nuevo navío o las nuevas aeronaves permiten a Brasil expandir su área de actuación o acceder a regiones anteriormente inaccesibles. Sin embargo, dicha evolución marca una reafirmación de la apuesta histórica del país en la región y va orientada a garantizar una alta calidad de las condiciones de trabajo para las investigaciones científicas que allí se desarrollan; asegurando a su vez un alto nivel de seguridad de sus operaciones y un desempeño operativo y ambiental acorde con los más altos estándares internacionales, cuestión que siempre ha sido considerada por Brasil como de fundamental importancia, y que permite recuperar el desempeño ejemplar ${ }^{11}$ que mantuviera por treinta años hasta el desafortunado incendio de su estación ${ }^{12}$.

Habiendo revisado, hasta aquí, cuál es el capital físico de infraestructura con el que Brasil cuenta para sus acciones en el continente blanco, pasaremos ahora a analizar la estructura institucional disponible y como ello impacta en su posición actual y sus posibilidades futuras.

\section{La Política Antártica Brasileña (POLANTAR) y su estructura institucional: evolución, características actuales y perspectivas futuras}

La elaboración de directrices para una política antártica de Brasil es anterior a la instalación de su Programa Antártico, pero posterior a su adhesión al Tratado Antártico ${ }^{13}$. Las primeras discusiones se iniciaron poco después de la adhesión brasileña en 1975, y concluyeron, en 1976, en la elaboración de un documento conteniendo orientaciones y directivas específicas para una Política Antártica Brasileña, denominada como POLANTAR, y que incluía el diseño institucional a adoptar (Política Nacional para Assuntos Antárticos, 1976). Dichas orientaciones se mantuvieron en sigilo, siendo revisadas y reformuladas en forma de decreto en 1987 (Decreto 94.401/87). La POLANTAR definida por decreto introdujo algunas modificaciones, pero mantuvo en esencia las orientaciones y el diseño institucional previstos en el documento de

\footnotetext{
${ }^{11}$ Siguiendo a Villa (2004, pp.130-132), para la organización Greenpeace, en su informe de 1988, apenas Brasil, Italia y Polonia merecieron reconocimiento por su desempeño ambiental, mientras que los otros países incumplían ampliamente las regulaciones ambientales. Si bien es muy probable que dicha situación haya cambiado substancialmente luego de la firma del Protocolo para la Protección del Medio Ambiente, es notorio que Brasil ya seguía una política de estricta observancia de las regulaciones ambientales antes de la firma del citado Protocolo.

${ }^{12}$ Sobre la importancia de la cuestión ambiental al interior de la Política Antártica de Brasil, ver: Cardone (2015).

${ }^{13}$ Brasil adhirió al Tratado Antártico por medio de notificación al Gobierno de los Estados Unidos como depositario del Tratado en 1975. Su primer programa antártico, denominado OPERANTAR I tuvo lugar en la temporada 1982-1983, lo que le permitió su ingreso como Miembro Consultivo del Tratado Antártico en 1983.
} 
1976. Esas mismas orientaciones de 1987 son las que permanecen vigente hoy en día, incluso cuando muchas de sus directivas se basan en las discusiones surgidas a partir del aprovechamiento de los recursos naturales, particularmente minerales, antes de la firma y ratificación del Protocolo al Tratado Antártico para la Protección del Medioambiente (conocido habitualmente como Protocolo de Madrid) $)^{14}$.

La POLANTAR definió, desde sus orígenes, la estructura institucional que Brasil debería adoptar en lo ateniente a su participación antártica. Desde el comienzo, Brasil planteó la necesidad de minimizar la estructura a fin de maximizar la economía de recursos, pero manteniendo un nivel de participación que le permitiese ser parte activa del régimen antártico. Para ello, se propuso que la estructura institucional para Antártida no sería específica, sino que usufructuaría de los recursos disponibles en otros ministerios. Inclusive la posibilidad de creación de un Instituto Antártico Brasileño fue prevista en 1976, pero acabó no materializándose ${ }^{15}$. Finalmente, se optaría por colocar bajo la Comisión Interministerial para los Recursos del Mar (CIRM) la responsabilidad general del programa.

En términos formales, el PROANTAR cuenta con dos comisiones asesoras, la Comisión Nacional para Asuntos Antárticos (CONANTAR), órgano interministerial bajo dirección del Ministerio de Relaciones Exteriores, previsto desde 1976 junto con el primer diseño institucional establecido por la POLANTAR, que debe asesorar a la Presidencia en la formulación y ejecución del PROANTAR; y el Comité Nacional de Investigaciones Antárticas (CONAPA), instituido en 1996 y bajo la dirección del Ministerio de Ciencia, Tecnología e Innovación. Sin embargo, tanto la CONANTAR como la CONAPA fueron disueltas por un Decreto Presidencial de 2019 (Decreto No 9.759/2019), que buscaba simplificar la estructura administrativa del Estado, pero que no previó la absorción de las funciones de los órganos extintos.

A pesar de que la CONANTAR debería ser la principal interface política, el análisis histórico de su trayectoria muestra que su relevancia ha sido limitada. Esto se revela en que la misma no había sido constituida cuando fue lanzada la primera campaña antártica brasileña, y que sus actividades fueron suspendidas entre 1995 y 2006 y, nuevamente, entre 2009 y 2014, sin que ello significase ningún tipo de interrupción en las actividades científicas del programa (Ferreira, 2009, pp.133, 149; Ata da 186 Sessão Ordinária, p. 14). Tal situación ha resultado aún más marcada a partir de su extinción el pasado año, y la asunción por parte de la CIRM de la responsabilidad que antes le era asignada. En respuesta a dicha situación, la CIRM resolvió, en julio de 2020, la creación de un "Grupo Técnico POLANTAR" el cual estará dedicado a la revisión de la Política Antártica Brasileña vigente con el objeto de mejorarla y actualizarla, incluyendo las consideraciones que responden a los cambios fundamentales que ha experimentado el régimen antártico desde la última versión vigente (Resolução No 16/2020). Por su parte, la CONAPA que fuera extinguida por el mismo decreto que la CONANTAR, ha

\footnotetext{
${ }^{14}$ Inclusive, puede afirmarse que su elaboración respondió al avance de las discusiones sobre recursos minerales que llevarían, ese mismo año, a la firma de la Convención para la Reglamentación de las Actividades sobre Recursos Minerales Antárticos (conocida habitualmente como Convención de Wellington). Al año siguiente, Francia y Australia anunciarían la no ratificación de dicha convención, imposibilitando su entrada en vigencia. Finalmente, dicha reglamentación sería reemplazada por la prohibición de actividades minerales no orientadas a la investigación científica impuesta por el Protocolo de Protección de Madrid.

${ }^{15}$ Sobre la historia del involucramiento brasileño con la Antártida, ver: Azambuja (2005); Borges (1959); Capozoli (2001); Ferreira (2009); Menezes (1982); Ramalhete (1975). Un trabajo comprehensivo, en inglés, sobre la historia del involucramiento brasileño en la Antártida puede encontrarse en: Cardone, I. (2021, en prensa). The Antarctic Politics of Brazil.
} 
tenido un papel limitado pero relevante en lo que se refiere a la estructuración de un programa científico consistente, y ha conseguido una decisión favorable para su reconstitución ${ }^{16}$.

Así establecido, el PROANTAR es encabezado por el secretario de la CIRM, que gerencia el programa, articulando con los ministerios: de Relaciones Exteriores, de Ciencia, Tecnología e Innovación, de Medio Ambiente, de Turismo, de Educación y Deportes; y con la Secretaría de Agricultura y Pesca y del Consejo Nacional de Investigaciones (CNPq); además de la colaboración brindada por Petrobrás en el abastecimiento de combustible, y Oi para la provisión del servicio de telecomunicaciones ${ }^{17}$. La Secretaría de la CIRM, a través de una subcomisión para el PROANTAR, coordina el accionar de tres grupos: el Grupo de Evaluación Ambiental $(\mathrm{GAAm})^{18}$, cuya principal responsabilidad recae sobre el Ministerio de Medio Ambiente; el Grupo de Asesoramiento $(\mathrm{GA})^{19}$, coordinado por el Ministerio de Ciencia, Tecnología e Innovación; y el Grupo de Operaciones $(\mathrm{GO})^{20}$, coordinado por la Secretaría de la CIRM.

La responsabilidad científica del programa está bajo la CONAPA, quien establece las prioridades y criterios para la selección de proyectos a ser desarrollados como parte del PROANTAR. La creación de tal Comité, en 1996, respondió a la evolución de la estructura institucional del programa brasileño a la luz de los cambios tanto en la política interna brasileña, como en la evolución del régimen antártico ${ }^{21}$. Originalmente, la postulación y selección de los proyectos de investigación científicos a ser desarrollados en la Antártida estaban a cargo de la Marina, a través de la CIRM. El proceso de democratización brasileño junto con el mayor énfasis dado al aspecto científico por sobre el aprovechamiento de los recursos naturales (especialmente minerales) a partir de la caída del acuerdo de explotación mineral y la firma del Protocolo al Tratado Antártico para la Protección del Medio Ambiente desplazaron la centralidad que las investigaciones vinculadas con el potencial mineral tuvieron anteriormente, haciendo que, a partir de 1991, la selección de los proyectos quedase bajo responsabilidad del CNPq (Avaliação preliminar do Programa Antártico Brasileiro, 2006). La conformación de la CONAPA institucionalizó dicha situación, siendo creada un año después de la ratificación del Protocolo de Madrid por parte del Congreso brasileño ${ }^{22}$.

\footnotetext{
${ }^{16}$ La noticia sobre su reconstitución fue obtenida a partir de una entrevista del autor con el Dr. Jefferson Simões (UFRGS, SCAR), 11 septiembre de 2020.

17 Datos extraídos del portal web oficial del PROANTAR: https://proantar.com.br/page/estrutura-doprograma-antartico-brasileiro-1574188044. Consulta: 5 de septiembre de 2020

${ }^{18}$ El GAAm es coordinado por el Ministerio del Medio Ambiente, y compuesto por representantes del Ministerio de Relaciones Exteriores, del Ministerio de Ciencia, Tecnología e Innovación, de la SeCIRM, del Instituto Brasileño de Medio Ambiente y Recursos Naturales Renovables (IBAMA), del CNPq, y un representante de la comunidad científica para cada una de las áreas que componen el PROANTAR (Ciencias Físicas, Geociencias y Ciencias de la Vida). Ver: Resolución 15, de 30 de julho de 2020 de la SeCIRM.

19 El GA es coordinado por el Ministerio de Ciencia, Tecnología e Innovación, y cuenta con representantes del Ministerio de Medio Ambiente, de la SeCIRM y del CNPq. Ver: Resolución 15, de 30 de julio de 2020 de la SeCIRM.

20 El GO es coordinado por la SeCIRM e integrado por representantes del Ministerio de Ciencia, Tecnología e Innovación, del Ministerio de Medio Ambiente, del CNPq, del Navio de Apoyo Ocenanográfico Ary Rongel y del Navio Polar Almirante Maximiano. Ver: Resolución 15, de 30 de julio de 2020 de la SeCIRM.

${ }^{21}$ Existió con anterioridad una comisión de investigación (CNPA) al interior del CNPq. La misma fue creada en 1984 para representar a Brasil internacionalmente dentro del Comité Científico de Investigaciones Antárticas (SCAR). Sin embargo, dicha comisión era presidida por el secretario de la CIRM y no adquirió el carácter de relevancia que tendría la CONAPA a partir de su constitución.

${ }^{22}$ Decreto Legislativo $\mathrm{N}^{\mathrm{o}}$ 88, del 6 de junio de 1995. Su ratificación definitiva se daría en 1998 por Decreto $\mathrm{N}^{\mathrm{o}} 2.742$, del 20 de agosto.
} 
Sin embargo, fue solo en la primera década del siglo XXI que el aspecto científico adquirió una mayor relevancia a partir del involucramiento del Ministerio de Medio Ambiente y la posterior creación de los Institutos Nacionales de Ciencia y Tecnología ligados a la investigación antártica, coincidiendo con una fuerte apuesta en la participación brasileña del programa del $4^{\text {to }}$ Año Polar Internacional. Entre los años 2002 y 2009, los llamados a proyectos de investigación experimentaron importantes cambios. El primero de estos cambios fue la articulación de proyectos integrados a través de las llamadas redes de investigación, que buscaban articular diferentes grupos, instituciones y disciplinas en torno de dos temáticas marco: los impactos ambientales globales y la Antártida; y los efectos de la acción humana en la Isla 25 de Mayo (Rey Jorge/Waterloo). Esto fue acompañado posteriormente por la articulación de llamadas integradas trianuales por parte del programa. Con anterioridad, los proyectos eran presentados, en un primer momento, bajo invitación de la CIRM y, en un segundo momento, a partir de 1991, por llamados abiertos anuales por parte del CNPq (Avaliação preliminar do Programa Antártico Brasileiro, 2006). A partir de las redes el programa pasó a tener dos componentes: el llamado a proyectos inducidos, es decir sobre temáticas predefinidas; y el llamado espontáneo, es decir sobre otras temáticas, incluyendo, a partir de 2009, a las humanidades y ciencias sociales dentro de las áreas a ser financiadas.

La transición del llamado a proyectos individuales a un programa integrado, significó la superación de la visión de un programa científico timoneado por individuos a un programa de carácter verdaderamente nacional y orientado a una coordinación con las metas definidas por el Comité Científico para la Investigación Antártica (SCAR, por sus siglas en inglés). Como resultado de un llamado realizado en 2008 para la creación de institutos de investigación ${ }^{23}$, fueron creados dos institutos, el Instituto Nacional de Ciência e Tecnologia Antártico de Pesquisas Ambientais, con sede en la Universidad Federal de Rio de Janeiro y hoy extinto; y el Instituto Nacional de Ciência e Tecnologia da Criosfera, con sede en la Universidad Federal de Rio Grande del Sur. La creación de dichos institutos se constituyó en un primer paso para institucionalizar ámbitos científicos específicamente antárticos (o polares en el caso del segundo), creando una estructura más descentralizada que la existente en otros países como Argentina o Chile.

En la actualidad, el Ministerio de Ciencia, Tecnología e Innovación, en conjunto con el CNPq, realizan la llamada a proyectos y el análisis de mérito científico; pasando luego a la evaluación y análisis de impacto ambiental (en cumplimiento de lo previsto por el Protocolo de Madrid), a cargo del Ministerio de Medio Ambiente. Luego, la Secretaría de la CIRM evalúa la factibilidad operativa en función de los recursos logísticos y presupuestarios disponibles. Por último, el Directorio Ejecutivo del CNPq evalúa los proyectos aprobados, emitiendo un dictamen, una vez considerados los límites presupuestarios de cada llamado.

En cuanto a la participación brasileña en los foros internacionales antárticos, esta está a cargo del Ministerio de Relaciones Exteriores, a través de su Dirección de Mar, Antártida y Espacio Exterior (DMAE). La agrupación de las temáticas de Mar, Antártida y Espacio Exterior, junto con la existencia de una estructura limitada y la carencia de especialistas de renombre, parecen indicar una importancia relativamente baja al interior de las cuestiones de Política Exterior. Ello explicaria, al menos parcialmente, la relativa modesta participación de Brasil en las Reuniones Consultivas del Tratado Antártico y el Comité de Protección Ambiental, aunque dicho desempeño no resulta diferente del de otros países consultivos no pertenecientes al grupo de los signatarios originales del Tratado (Sánchez, 2016) 24

\footnotetext{
${ }^{23}$ Dicho llamado no fue específico para cuestiones antárticas, sino que respondió a una iniciativa general para la promoción de institutos de ciencia, tecnología e innovación.

${ }^{24}$ En este sentido, es de destacar que la variable de haber establecido un reclamo territorial o una reserva de derechos explícita antes de la firma del Tratado permanecen como la variable más relevante en términos de la participación. Así, el llamado Grupo de los 7+2 (los siete reclamantes: Argentina,
} 
Como puede notarse, la totalidad del diseño institucional brasileño gira en torno a la cuestión operativa y logística, con la Marina de Brasil ocupando un lugar central. La extinción de la CONANTAR ciertamente ha reforzado esa centralidad de la Marina y, por la forma que ha adoptado la revisión de la POLANTAR, no ha de esperarse que dicha situación cambie considerablemente en el futuro cercano. La revisión de la POLANTAR ciertamente proporciona una oportunidad para repensar y reformular tanto los principios rectores de la intervención brasileña en la Antártida, cuanto la estructura institucional pensada para dar soporte a dicha intervención. Sin embargo, la mecánica de revisión centrada en la Marina brasileña y la falta de una discusión más amplia y participativa al respecto hacen pensar que dicho rediseño está lejos de presentar un replanteo profundo al respecto de los objetivos y políticas del país en la Antártida.

El papel del Ministerio de Ciencia, Tecnología e Innovación y del CNPq resultan relevantes en lo que se refiere a la definición de los programas científicos a través de la CONAPA, pero ciertamente aparece en un lugar subordinado dentro de la estructura institucional del PROANTAR en su conjunto. Por su parte, el Ministerio de Relaciones Exteriores actúa casi exclusivamente en su rol de representación al interior del régimen internacional antártico, con un accionar reducido en otros ámbitos.

A su vez, la ausencia de una estructura institucional específica brinda, por un lado, una relativa flexibilidad que facilita la adaptación del programa a las necesidades y posibilidades de cada momento. Sin embargo, por otro lado, tal ausencia determina una cierta fragilidad a las vicisitudes políticas y económicas, y reduce las posibilidades de influencia que los actores vinculados a dicho programa podrían ejercer de contar con un esquema institucional más centralizado. Además de ello, dicha carencia resulta también en la falta de una memoria institucional, histórica y documental que pueda constituirse en marco de una identidad vinculada al patrimonio histórico de la presencia brasileña en la Antártida.

Hasta aquí hemos analizado la disponibilidad de infraestructuras materiales e institucionales para Brasil en sus intervenciones antárticas. Pasaremos a continuación a evaluar las condiciones y perspectivas futuras de lo que es, históricamente y en base a lo determinado por el Tratado Antártico, la principal actividad humana en la región, esto es, la investigación científica.

\section{La investigación brasileña en la Antártida: fortalezas y debilidades de la espina dorsal del Programa Antártico Brasileño}

El programa científico antártico de Brasil evolucionó de una actividad subordinada a sus intereses de participar del régimen antártico a un programa altamente especializado, estructurado y articulado en función de metas científicas claramente definidas. Dicha evolución respondió, por un lado y particularmente en los albores del programa, a la articulación de acciones estatales orientadas a formar académicos especializados en investigación antártica ${ }^{25}$, pero también a la evolución del propio régimen antártico y el accionar individual de algunos académicos y militares brasileños. Si bien ello determinó un alto grado de flexibilidad y adaptabilidad de las investigaciones antárticas de ese país, también generó una situación de gran dependencia de individuos que operaron $-\mathrm{y}$, en gran medida, aún operan - como fuerzas centrípetas de los proyectos de investigación antártica.

Australia, Chile, Francia, Noruega, Nueva Zelandia y Reino Unido; sumados a los dos que establecieron explícitamente su reserva de derechos: Estados Unidos y la Unión Soviética, hoy Rusia) continúa siendo el grupo más activo, aunque ciertos patrones comienzan a cambiar paulatinamente (Sánchez, 2016).

${ }^{25}$ En el comienzo del programa antártico brasileño el CNPq, por pedido de la CIRM, lanzó un llamado a 20 becas para la formación de académicos en ciencias polares. Información obtenida a partir de una entrevista realizada por el autor con el Dr. Jefferson Simões (UFRGS, SCAR), 11 de septiembre de 2015. 
En los albores del PROANTAR, la investigación científica tuvo un carácter altamente personalista. Investigadores de alto perfil y con buena trayectoria orientaron sus investigaciones al nuevo terreno que proporcionaba oportunidades de relieve nacional e internacional. La definición del programa por invitación hacía que los proyectos llevados a cabo en la Antártida fueran altamente direccionados, privando parte de la iniciativa al ámbito científico. Cuando, en 1991, como fue visto anteriormente, la responsabilidad por el programa científico fue transferida al CNPq, ello no cambió significativamente el perfil de las investigaciones ni de los grupos que actuaban allí. Todavía más, dicha transferencia no fue realizada junto con los recursos económicos necesarios para su implementación, dejando en algunos momentos al programa científico al borde del colapso ${ }^{26}$.

Tal situación comenzó a cambiar recién a partir de los primeros años del siglo XXI con el involucramiento del Ministerio de Medio Ambiente que, en 2002, promovió la creación de dos redes de investigación en la búsqueda de articular los proyectos en programas amplios que incluyeran un gran número de instituciones y estuvieran vinculados con los de otros países, particularmente en lo que se refiere al impacto ambiental. Las dificultades financieras con las cuales el programa venía contando llevaron a un grupo de diputados a promover la creación de un Frente Parlamentario de apoyo al PROANTAR en 2003 (Diârio da Câmara dos Deputados, 11 Dic 2003, p. 67109-67111), que fue renovado en sucesivas legislaturas. En 2005 y continuando con el esfuerzo de estructuración de un programa con metas y objetivos definidos, el Ministerio de Ciencia y Tecnología de aquella época instituyó el grupo de trabajo "Agenda Antártica", orientado a establecer un plan de trabajo quinquenal (Portaria MCT No 397/2005). La articulación de la "Agenda Antártica" junto con el apoyo político obtenido a partir del Frente Parlamentario de apoyo al PROANTAR le permitió a Brasil incorporarse al Año Polar Internacional de 2007-2009 (Gandra, 2013, pp. 113-116) con un programa consistente y de alcance considerable.

Toda esa trayectoria se consolidaría, en 2008, con la creación de los INCTs descriptos en la sección anterior, que independizaron los objetivos de una ciencia polar y antártica de las iniciativas individuales, e incrementaron considerablemente la cantidad de recursos disponibles para la investigación ${ }^{27}$; evolucionando desde la realización de llamados integrados de propuestas de investigación desde 2002 hasta la generación de un plan estratégico de ciencia antártica, denominado "Ciência Antártica para o Brasil: Plano de Ação 2013-2022" (2013), elaborado por la CONAPA. Dicho plan estratégico definió cinco programas, orientados a explorar las conexiones entre el continente antártico y América del Sur, e incluyen estudios sobre la criósfera; las dinámicas de la alta atmósfera en la Antártida; y la influencia del cambio climático sobre los ecosistemas antárticos y sobre el Océano Austral ${ }^{28}$. Llama la atención, sin embargo, lo que es definido en dicho programa como su misión central, la cual se muestra como heredera del origen instrumental de la ciencia para la política antártica de Brasil, al definir como tal: "Desenvolver un programa de investigación de excelencia sobre la región antártica y sus conexiones con el Océano Atlántico y América del Sur, contribuyendo a garantizar la permanencia de Brasil como miembro consultivo del Tratado Antártico" (Simões et al., 2013 , p. 3$)^{29}$. También, de relevancia resulta la ausencia de un programa específico orientado al desarrollo de las ciencias sociales y humanidades antárticas.

\footnotetext{
${ }^{26}$ Idem.

${ }^{27}$ Esto se da porque dichos Institutos cuentan con un presupuesto proprio, de base plurianual, y que complementa los recursos disponibles a partir de los llamados específicos del PROANTAR.

28 Específicamente, los programas son: 1) El papel de la criósfera en el sistema terrestre y las interacciones con América del Sur; 2) Efectos del cambio climático en la bio-complejidad de los ecosistemas antárticos y su conexión con América del Sur; 3) Cambio Climático y Océano Austral; 4) Geodinámica e historia geológica de la Antártida y sus relaciones con América del Sur; 5) Dinámica de la alta atmósfera en la Antártida, interacciones con el geoespacio y conexiones con América del Sur.

${ }^{29}$ Énfasis agregado, traducción propia.
} 
En términos del incidente que resultó en la destrucción casi completa de la EACF, en 2012, Brasil ha conseguido mantener su producción científica antártica en un alto nivel de productividad e impacto, dando continuidad a la gran mayoría de sus programas e incorporando nuevas líneas de investigación ${ }^{30}$. Sin embargo, el programa también ha experimentado numerosas dificultades financieras y algunas pérdidas significativas, como es la desarticulación del INCT Antártico de Pesquisas Ambientais y la no obtención de financiamiento por parte de ningún proyecto de humanidades y ciencias en el último llamado del PROANTAR (2018).

En términos generales, el PROANTAR no ha contado con una previsibilidad financiera que permita inferir la continuidad de las investigaciones, teniendo que articular políticamente a la fecha de vencimiento de cada llamado - o incluso anticipadamente - para que nuevos recursos sean colocados a disposición a partir de enmiendas parlamentarias, y gracias al accionar de individuos influyentes, grupos políticos y de la propia Marina brasileña ${ }^{31}$. Para tomar dimensión de las diferencias existentes entre el financiamiento ofrecido para la infraestructura física y aquel disponible específicamente para los proyectos de investigación, la reconstrucción de la EACF ha demandado una inversión de, aproximadamente, unos 100 millones de dólares americanos (550 millones de reales a la cotización actual), mientras que los programas científicos han recibido aproximadamente unos 3,3 millones de dólares americanos (18 millones de reales) para un período de tres años (2019-2022, extendido ahora por un año más por el cancelamiento de la operación 2020-2021).

A pesar de las limitaciones que la ciencia antártica brasileña ha sufrido a lo largo del tiempo, es de destacar que ésta ha conseguido establecer un patrón de desempeño que le ha proporcionado una relevante visibilidad e influencia internacional. A pocos años de su ingreso en la actividad antártica y menos de dos años luego de su incorporación al SCAR, el geologista Antônio Rocha-Campos fue elegido secretario del SCAR, cargo que ocuparía entre 1986 y 1992; y luego presidente de esa misma institución, entre 1994 y $1998^{32}$. A ello se sumaría la elección de la bióloga Edith Fanta como presidenta del Comité Científico de la Comisión para la Conservación de Recursos Vivos Marinos Antárticos (CCRVMA), desde 2004 hasta su prematuro fallecimiento en 2008, y la elección del glaciólogo Jefferson Simões como vicepresidente del SCAR desde 2016 hasta la actualidad.

Lo analizado anteriormente determina que, a pesar de los considerables logros y avances positivos descriptos aquí, el programa científico de Brasil en la Antártida presente dos grandes debilidades. La primera se refiere al alcance del programa científico, al menos en su dimensión estratégica, y está dada por la ausencia de un programa dedicado a las ciencias sociales, incluso frente a la presencia de un grupo con marcada presencia internacional e impacto y la labor realizada de modo aislado por diversos investigadores brasileños. La segunda, y que afecta a la totalidad del programa antártico, tiene que ver con las limitaciones de financiamiento del programa, las cuales han sido una constante en el pasado y se proyecta también hacia el futuro, cuestionando la centralidad de la actividad científica al interior del PROANTAR.

En términos de la primera limitación, el PROANTAR no ha definido un programa para las humanidades y ciencias sociales, quedando esta área completamente relegada, incluso frente

\footnotetext{
${ }^{30}$ Esto ha sido posible, en gran medida, gracias a la colaboración brindada por otros países, entre los cuales la colaboración argentina y chilena ha sido fundamental. Al mismo tiempo, tal constatación ha relativizado la importancia que la EACF tiene en términos de su función como base del trabajo científico brasileño.

31 Para un breve análisis de la evolución financiera del programa y la necesidad de enmiendas parlamentarias, ver: Cardone (2014). Un análisis sobre la situación crítica del programa en 2018 puede verse en: Simões (2018).

${ }^{32}$ Hasta el día de hoy Rocha-Campos ha sido el único latinoamericano en ocupar el lugar de presidente del SCAR.
} 
a la creciente importancia que ha adquirido al interior del propio Comité Científico para la Investigación Antártica ${ }^{33}$. Ello, aún cuando un grupo ya consolidado de la Universidad Federal de Minas Gerais (UFMG), en colaboración con instituciones e investigadores de diversos países, viene realizando, desde 2009, un programa de investigaciones arqueológicas vinculado a la región antártica y sub-antártica, y el cuál ha quedado fuera de los proyectos financiados en el último llamado del PROANTAR, poniendo en riesgo la continuidad de la investigación. Cabe aclarar que dicha exclusión se da en el marco de un concurso en el cual la composición del jurado no contó con ningún especialista del área de humanidades y ciencias sociales, y que ninguna de las propuestas de dicha área obtuvo financiamiento ${ }^{34}$. Ello puede explicar, en parte, porque las humanidades y ciencias sociales antárticas no han evolucionado en Brasil, quedando limitadas a trabajos puntuales en algún momento de la carrera académica de diversos individuos y sin constituir, a excepción del citado caso de la UFMG, grupos estables con programas de largo alcance.

Por otro lado, en lo que se refiere a las dificultades financieras, las debilidades del diseño institucional brasileño para la Antártida determinan que no exista una interface permanente que ayude a constituir un esfuerzo continuado en el financiamiento a investigación antártica -tal vez con la única excepción de los INCTs. La ausencia de compromisos de largo plazo hace que esfuerzos de cabildeo deban ser realizados continuamente, dependiendo en su efectividad del momento político y económico de la coyuntura nacional. A esto debe sumársele la situación actual, iniciada luego de la destitución forzada de la presidenta Dilma Rousseff y su reemplazo por el vicepresidente Michel Temer, pero claramente acentuada luego de la asunción a la presidencia por parte de Jair Bolsonaro, de una política de Estado fuertemente contraria a la investigación científica y que ha planteado una fuerte desinversión, retracción y ataque al sector. Dicha política no solo compromete las perspectivas de financiamiento del programa antártico en sí, sino que también impacta indirectamente por sus efectos sobre el sistema científico en general, al limitar el capital de recursos humanos disponibles y las perspectivas de carrera para los académicos ${ }^{35}$. Ello determina que, en el futuro, el financiamiento para el programa científico del PROANTAR probablemente dependerá más de la importancia que le sea atribuida a la presencia brasileña en la región y la influencia que pueda ejercer la Marina brasileña, que a un reconocimiento político de la importancia de la ciencia antártica como un factor de desarrollo del país.

\section{Conclusiones: un programa consolidado pero de expansión limitada}

A pesar de que la fuerte inversión en infraestructura física pueda sugerir una apuesta renovada por parte de Brasil en la Antártida, el análisis presentado aquí sugiere que dicha apuesta se trata más de una acción tendiente a reforzar la posición ya ganada por Brasil en dicha región, que a perseguir un nuevo posicionamiento dentro del continente. En particular, resulta revelador que la fuerte inversión brasileña se centre en la renovación y modernización de las capacidades preexistentes, y no en la expansión del área de actuación o la adquisición de nuevas

\footnotetext{
${ }^{33}$ Esto se evidencia a partir de la constitución de los antiguos grupos de expertos en Humanidades y Ciencias Sociales del SCAR, como Comité Permanente (SC-HASS/SCAR), lo que significa un reconocimiento a la importancia que dichas cuestiones están adquiriendo al interior del corpus académico sobre Antártida en el ámbito internacional.

34 Información obtenida a partir de una entrevista del autor con el Dr. Andrés Zarankin (UFMG), realizada el 9 de septiembre de 2020.

${ }^{35}$ En este sentido, uno de los efectos más claros está dado por las suspensiones y cortes de los programas de becas los cuales han retraído significativamente las posibilidades de realización de estudios de postgrado y, muy especialmente, las alternativas de inserción laboral temprana de los recién doctores, privando a los laboratorios de una muy necesaria mano de obra especializada.
} 
capacidades operativas o el desarrollo de un conocimiento más desarrollado sobre la realidad política e institucional del continente blanco.

Al mismo tiempo, la diferencia existente entre las formas, dimensiones y facilidades que adquiere la inversión en infraestructura física logística se contrapone fuertemente con las dificultades y limitaciones con las que cuenta la financiación específica para la realización de investigaciones científicas. Ello es indicativo de las diferencias existentes en la prioridad brindada a uno y otro aspecto, así como a las diferencias en el acceso a recursos financieros entre las Fuerzas Armadas - particularmente, en el caso que nos ocupa, la Marina brasileña- y el Ministerio de Ciencia, Tecnología e Innovación. En ese sentido, la visibilidad e influencia obtenida a nivel internacional por la ciencia antártica brasileña, manifiesta a partir de destaques como la elección del Dr. Jefferson Simões como vicepresidente del SCAR, no se traduce en una mayor prioridad a nivel nacional.

En términos institucionales, la estructura brasileña muestra, por un lado, una relativa flexibilidad y economía de recursos pero, por otro, importantes limitaciones. En primer lugar, la disolución de la CONANTAR resulta en la institucionalización del papel dominante de la Marina brasileña, particularmente como articulador político del programa, colocando a dicha institución, incluso, como eje de la revisión de los principios rectores de su política antártica. No se entrevé que la nueva POLANTAR indique nuevas orientaciones o que incluya otras fuerzas, tales como al Ejército brasileño, de modo más activo. Ello puede explicar el énfasis fundamentalmente marítimo y costero del programa brasileño y la necesidad de recurrir a la asistencia de otros programas o contratistas privados para las actividades que implican adentrarse al interior del continente.

Por otro lado, la permanencia de un carácter periférico de la cuestión al interior de Itamaraty y la falta de una institución antártica centralizada y de centros especializados en investigación de humanidades y ciencias sociales limita las capacidades de conocimiento y de actuación informada por parte de Brasil en las interfaces políticas, inclusive en lo que se refiere a la asistencia a los tomadores de decisiones. Así, no es de esperar que Brasil consiga formular una política antártica que cuente con un alto grado de iniciativa, en el sentido de conseguir articular acciones innovadoras y tendientes a cambios substantivos al interior del régimen antártico.

En la cuestión científica específicamente, resulta limitador la falta de inclusión de estudios sobre humanidades y ciencias sociales al interior de los programas estratégicos de ciencia antártica, así como la permanencia de una visión de la ciencia como garante de la participación política de Brasil en el régimen antártico y no siguiendo su fuerza y utilidad intrínsecas. Reconociendo así su rol instrumental al interior de la política antártica de tal país, la ciencia antártica brasileña pierde la oportunidad para definir un genuino interés científico en la generación de conocimiento, el cual le permitiría avanzar en una estructuración más sustentable en el tiempo y vinculada con los intereses de su competencia.

Ello explica, a su vez, porque la ciencia antártica brasileña debe atravesar cada pocos años una situación crítica de imprevisibilidad de financiamiento, dependiendo en gran medida del circuito político y la influencia de ciertos grupos con interés en el programa. Cuando se compara el volumen de financiamiento de las actividades científicas durante los últimos años con lo invertido en la renovación de la EAFC o del parque de navíos y aeronaves, queda claro que la supuesta prioridad brindada a la ciencia no se traduce en la inversión para sus principales actores, esto es, los científicos e instituciones científicas, sino que está fuertemente anquilosada a una visión más tradicional sobre la relevancia de la presencia física como factor de expresión máximo del interés y accionar en el continente. Sumado a ello, la actual política de fuerte reducción de la inversión en ciencia y los ataques directos e indirectos al sector parecen comprometer las perspectivas a futuro para dicha actividad. 
Por esto último, podemos concluir que el programa antártico brasileño sigue claramente vinculado a una visión que coloca el énfasis en la importancia de la presencia física en la Antártida, quedando la investigación científica vinculada a las necesidades impuestas por el Tratado Antártico para participar en la toma de decisiones, más de que un reconocimiento genuino de su utilidad e interés específicos. En ese sentido, la nueva EACF constituye la máxima expresión de ese compromiso renovado con la presencia física en Antártica, tanto por la inversión demandada, como por el simbolismo que implica en términos de su diseño innovador y tecnología de punta. Así, Brasil continúa concentrando sus esfuerzos en establecer una presencia física y logística de punta, más que expandir o proyectar sus capacidades hacia nuevos escenarios de la región. Ello responde a una opción estratégica que prioriza la calidad sobre la extensión en cuanto a infraestructura física, y que es consistente con la trayectoria histórica de Brasil en el continente. Por otro lado, en lo que se refiere a la inversión en ciencia antártica, el financiamiento dado a la misma no acompaña el incremento brindado para la parte logística y operativa, privando al programa antártico brasileño de hacer uso pleno de sus potencialidades.

Por lo visto a lo largo de este trabajo, es de esperar que Brasil continúe en gran medida con la misma trayectoria que ha mantenido hasta aquí, consolidando su perfil dentro de los límites ya establecidos, pero haciendo gran énfasis en su responsabilidad ambiental y seguridad operativa. A pesar de que tal opción se presenta como una elección acertada y adecuada a los actuales objetivos de Brasil en la Antártida, permitiendo el fortalecimiento de su programa científico de modo paulatino, las limitaciones experimentadas en términos del financiamiento a la actividad científica impiden el aprovechamiento pleno de sus potencialidades. Así, podemos concluir que Brasil continuará manteniendo su trayectoria de involucramiento antártico, pero no es de esperar que amplíe considerablemente sus operaciones e investigaciones, o reformule de modo significativo su papel en el continente blanco o en el régimen internacional creado para su administración.

\section{Referencias Bibliográficas}

Abdenur, A. E. \& Neto, D. M. (2014). Rising powers and Antarctica: Brazil's changing interests. Polar Journal, 4(1), pp. 12-27. https://doi.org/10.1080/2154896X.2014.913910

Avaliação preliminar do Programa Antártico Brasileiro (2006). Brasília

Azambuja, P. (2005). O sonho do aurora austral: como o Brasil chegou à Antártida. Balneário Camboriú: Magna Quies

Borges, D. R. S. (1959). Um brasileiro na Antártida; crônicas de uma viagem ao redor do mundo. São Paulo: Sociedade Geográfica Brasileira

Capozoli, U. (2001). Antártida: a última terra (3rd ed.). São Paulo: Editora da Universidade de São Paulo

Cardone, I. J. (2014). A Política Antártica Brasileira no século XXI: evolução de fatores estruturais. In Anais do Seminário Nacional Sociologia \& Política. Curitiba: UFPR

Cardone, I. J. (2015). As Posições Brasileiras No Sistema Do Tratado Antártico Com Ênfase Na Questão Ambiental. Universidade Federal de Parana. https://doi.org/http://hdl.handle.net/1884/38844

Child, J. (1988). Antarctica and South American Geopolitics: Frozen Lebensraum. New York: Praeger 
Colacrai, M. (1988). Cooperación: un desafio para las políticas antárticas latinoamericanas. Revista de Ciência Política, 31(2), pp. 41-49

Colacrai, M. (1989). Brasil: su política antártica y los espacios para una acción cooperativa con Argentina. Revista GEOSUR, 109-110, pp. 22-38.

Ferreira, F. R. G. (2009). O Sistema do Tratado da Antártica: evolução do regime e seu impacto na política externa brasileira. Brasilia: Fundação Alexandre de Gusmão

Gandra, R. M. (2009). O Brasil e a Antártida: ciência e geopolítica. Geografias, 05(2), pp. 6574. Recuperado de: http://www.jurua.com.br/shop item.asp?id=12348

Gandra, R. M. (2013). Geopolítica Antártica no Limiar do Século XXI: a definição de um projeto estratégico-científico para o Brasil na Antártida. Universidade Federal de Rio Grande do Sul

Lemmertz, H. (2015). A Ciência nos Confins da Terra: a arena transepistêmica da ciência antártica brasileira. Universidade Federal de Rio Grande do Sul. Recuperado de: http://weekly.cnbnews.com/news/article.html?no=124000

Mattos, L. F. de (2014). A Inclusão da Antártida no Conceito de Entorno Estratégico Brasileiro. Revista Da Escola de Guerra Naval, 20(1), pp. 165-192. Recuperado de: http://www.egn.mb/arquivos/revistaEgn/nova-revista/revista- 20n1/index.htm

Mattos, L. F. de (2015). O Brasil e a Adesão ao Tratado da Antártica: uma análise de política externa no governo Geisel. Universidade Federal Fluminense

Menezes, E. C. de (1982). Antartida e os Desafios do Futuro. Rio de Janeiro: Capemi Editora

Nascimento, C. D. (2007). O Programa Antártico Brasileiro PROANTAR: questões de gestão e representação da informação no contexto da produção científica. Universidade Federal Fluminense. Recuperado

de: http://www.redebim.dphdm.mar.mil.br/vinculos/000009/000009fd.pdf

Pereira, P. M. (2009). Questão Global e ação do Estado em uma perspectiva comparada: Brasil e Argentina. Universidade de Brasília

Ramalhete, C. (1975). A Antártica e o Brasil. Revista de Informação Legislativa, pp. 41-56

Sampaio, D. P., Cardone, I. J., \& Abdenur, A. E. (2017). A modest but intensifying power? Brazil, the Antarctic Treaty System and Antarctica. En K. Dodds, A. D. Hemmings, \& P. Roberts (Eds.). Handbook on the Politics of Antarctica (pp. 301-317). Cheltenham Northhampton: Edward Elgar Publishing

Sánchez, R. A. (2016). A brief analysis of countries' patterns of participation in the Antarctic Treaty Consultative Meetings (1998-2011); towards leveling the playing field ? Polar Record, 52(267), pp. 686-697. https://doi.org/10.1017/S0032247416000073

Simões, J. et al. (2013). Ciência Antártica para o Brasil: Um plano de ação para o período 2013-2022. Brasília

Villa, R. A. D. (2004). A Antártida no Sistema Internacional. São Paulo: Hucitec

\section{Leyes Decretos y Resoluciones de Órganos Públicos}

Brasil, Presidencia da República (11 Abr 2019). Decreto $N^{o}$ 9.759/2019 (Extingue e estabelece diretrizes, regras e limitações para colegiados da administração pública federal)

CIRM (2020a). Resolução $N^{o}$ 15/2020. Recuperado de: https://www.marinha.mil.br/secirm/sites/www.marinha.mil.br.secirm/files/documentos/at as/resolucao-15-2020.pdf. Consulta: 5 de septiembre de 2020 
CIRM (2020b). Resolução $N^{o}$ 16/2020. Recuperado de: https://www.marinha.mil.br/secirm/sites/www.marinha.mil.br.secirm/files/documentos/at as/resolucao-16-2020.pdf. Consulta: 5 de septiembre de 2020

Diârio da Câmara dos Deputados (11 Dic 2003). Disponible en: http://imagem.camara.gov.br/Imagem/d/pdf/DCD11DEZ2003.pdf. Consulta: 5 de septiembre de 2020

MCT (17 Jun 2005). Portaria MCT N $N^{o} 397 / 2005$

\section{Archivos Públicos}

Exposición de Motivos N016/76 (7 Jun 1976). BR/RJANEIRO/TT/0/JUS/PRO/0538. Arquivo Nacional (Brasil)

Política Nacional para Assuntos Antárticos - Directrizes Gerais (28 Oct 1976). BR/RJANEIRO/TT/0/JUS/PRO/0538. Arquivo Nacional (Brasil)

Ata da 186 Sessão Ordinária, 24 de abril de 2014. CIRM

\section{Artículos de Noticias}

Marinha do Brasil recebe segunda aeronave UH-17 para Operação "Antártica" (2020). Marinha do Brasil - Notícias. 10 de Julho Recuperado de: https://www.marinha.mil.br/noticias/marinha-do-brasil-recebe-segunda-aeronave-uh-17para-operacao-antartica. Consulta: 5 de septiembre de 2020

Mesquita, J. L. (2019). Pesquisas do Brasil na Antártica podem parar por motivos de cortes no orçamento. Mar sem Fim. 4 de Outubro. Recuperado de: https://marsemfim.com.br/pesquisas-do-brasil-na-antartica-podem-parar-por-cortes.

Consulta: 18 de octubre de 2019

Olivera, D. (2020). Projeto de novo Navio de Apoio Antártico segue em consulta. SINAVAL, 12 de Maio. Recuperado de: http://sinaval.org.br/2020/05/projeto-de-novo-navio-de-apoioantartico-segue-em-consulta/. Consulta: 23 de agosto de 2020

Silveira, E. (2019). Como Cortes do governo podem paralisar pesquisas do Brasil na Antártida. BBC News Brasil. 2 de Outubro. Recuperado de: https://www.bbc.com/portuguese/brasil-49887243. Consulta: 18 de octubre de 2019

\section{Bases de Datos y Otras Fuentes}

Banco Mundial (2019). Base de Datos sobre ranking de países según Producto Bruto Interno. Recuperado de: https://datacatalog.worldbank.org/dataset/gdp-ranking-ppp-based y https://datacatalog. worldbank.org/dataset/gdp-ranking. Consulta: 18 de Agosto de 2020

PROANTAR: https://proantar.com.br/

Secretaria da Comissão Interministerial para os Recursos do Mar: https://www.marinha.mil.br/secirm/

Secretaría del Tratado Antártico: https://ats.aq/index_s.html

Simões, J. (2018). Falta de recursos financeiros ameaça a continuidade da parte científica do Programa Antártico Brasileiro (PROANTAR) a partir de julho de 2018 - Análise de situação - Centro Polar e Climático/UFRGS. Recuperado de: http://www.diretodaciencia.com/wp-content/Anexos/Analise-Situacao-Proantar-2018.pdf. Consulta: 7 de enero de 2021 
Cuadernos de Política Exterior Argentina (Nueva Época), 133, junio 2021, pp. 29-46 ISSN 1852-7213 (edición en línea)

\section{Acrónimos y Siglas}

CIRM Comisión Interministerial para los Recursos del Mar (Brasil)

CNPq Comisión Nacional de Investigaciones (Brasil)

CONANTAR Comisión Nacional Antártica (Brasil)

CONAPA Comisión Nacional de Investigación Antártica (Brasil)

EACF Estación Antártica Comandante Ferraz (Brasil)

POLANTAR Política Antártica Brasileña

PROANTAR Programa Antártico Brasileño

SCAR Comité Científico para la Investigación Antártica (Internacional)

SeCIRM Secretaría de la Comisión Interministerial para los Recursos del Mar (Brasil) 